\title{
Conductas sexuales de riesgo en la era digital: análisis del fenómeno sexting en la población adulta joven española
}

\author{
Sexual risk behaviors in the digital age: analysis of the sexting \\ phenomenon in the young Spanish adult population
}

\author{
Carmen Rodríguez-Domínguez \\ Universidad de Huelva \\ Mercedes Durán Segura \\ Universidad de Sevilla
}

Recibido: 10/10/2018

Aceptado: 12/06/2019

\begin{abstract}
Prevention programs on risk behaviors through technologies are aimed primarily at children, but rarely focus on the older population. To promote this new approach, it is necessary to investigate the behavior of adults with technologies, especially when these behaviors can lead to unwanted public exposure of the individual privacy. Thus, the aim of this paper is to study the phenomenon of sexting or exchange of erotic-sexual contents through electronic means in a sample of 171 Spanish adults, comparing the results according to two age groups (18 - 26 years and 27 38 years). An associative-correlational study was carried out using an online questionnaire. The main results show a high participation in sexting, especially in the reception of sexual contents. A greater number of men than women send their own sexual images, being also men who receive this type of images more frequently. Participants younger than 26 are more involved than participants older than 26 in active sexting (to send) and passive sexting (to receive). Likewise, sexting is carried out with different profiles of people, although fundamentally with the partner, standing out among the most frequent motivations the intention to satisfy. The need to deepen the study of sexting in adults and to consider the potential risks of this practice in a non-consensual diffusion of erotic-sexual images is discussed. KEY WORDS: sexting, adults, gender, technologies, sexual behaviors.
\end{abstract}

\section{RESUMEN}

Los programas preventivos sobre conductas de riesgo a través de las tecnologías se dirigen fundamentalmente a menores de edad, pero escasamente se centran en la población mayor de edad. Para promover este nuevo enfoque es preciso investigar el comportamiento de adultos ante las tecnologías, especialmente cuando tales conductas pueden derivar en una exposición pública no deseada de la intimidad de la persona. Por ello, el objetivo de este trabajo es estudiar el fenómeno del sexting o intercambio de contenidos erótico-sexuales a través de medios electrónicos en una muestra de 171 adultos españoles, comparando los resultados según dos grupos de edad (18 - 26 años y 27 - 38 años). Se llevó a cabo un estudio asociativo-correlacional mediante cuestionario online. Los principales resultados muestran una alta participación en sexting, especialmente en la recepción de contenidos sexuales. Un mayor número de hombres que de mujeres envían imágenes propias de índole sexual, siendo también los hombres los que reciben con mayor asiduidad este tipo de imágenes. Los participantes menores de 26 años se involucran con más frecuencia que los mayores de 26 años en sexting activo (enviar) y sexting pasivo (recibir). Asimismo, se realiza sexting con diferentes perfiles de personas, aunque fundamentalmente con la pareja, destacándose entre las motivaciones más frecuentes la intención de satisfacer. Se discute la necesidad de profundizar en el estudio del sexting en adultos y de considerar los riesgos potenciales de esta práctica ante una difusión no consentida de las imágenes erótico-sexuales. PALABRAS CLAVE: sexting, adultos, género, tecnologías, comportamientos sexuales.

Dirección de correspondencia:

Carmen Rodríguez-Domínguez, Universidad de Huelva, Departamento de Psicología Clínica y Experimental. E-mail: mcarmen.rodriguez@dpces.uhu.es.

Mercedes Durán Segura, Universidad de Sevilla, Departamento de Psicología Social. 


\section{Introducción}

Los avances tecnológicos y la evolución cultural que ha supuesto en nuestra sociedad definen nuevas formas de comunicación e interacción interpersonal (Cornejo y Tapia, 2011). Entre estas nuevas formas relacionales se encuentra el sexting. Este término alude al intercambio de contenidos erótico-sexuales a través de tecnologías y medios informáticos, principalmente teléfonos móviles e Internet. Incluye el envío, recepción y reenvío de fotografías, vídeos o mensajes de texto sexualmente explícitos o implícitos (Alonso-Ruido, Rodríguez-Castro, Pérez-André y José-Magalhães, 2015; Hinduja y Patchin, 2012; Lenhart, 2009) producidos por el propio protagonista (McLaughlin, 2010; Pérez et al., 2011).

Se trata de un fenómeno de origen reciente (Cruz y Soriano, 2014) que comienza a ser asociado a un amplio rango de conductas de riesgo para la salud (para una revisión, Van Ouytsel, Walrave, Ponnet, Heirman, 2015). No obstante, hay expertos que consideran el sexting como una manifestación más de la sexualidad que no necesariamente se relaciona con conductas de riesgo (Ferguson, 2011; Gordon-Messer, Bauermeister, Grodzinski y Zimmerman, 2013) y que en el caso de adolescentes puede reflejar una forma de adaptar los elementos culturales y tecnológicos disponibles en su tiempo a las necesidades de expresión personal (Menjívar, 2010).

Los trabajos sobre sexting se centran en su mayoría en población adolescente (e.g., Dake, Price, Maziarz, y Ward, 2012; Durán y Rodríguez-Domínguez, 2017; Fajardo, Gordillo y Regalado, 2013; Gámez-Guadix, Santisteban y Resett, 2017; Rodríguez-Castro, Alonso-Ruido, González-Fernández, Lameiras-Fernández y Carrera-Fernández, 2017). Sin embargo, no solo los/as adolescentes están en contacto con las tecnologías de la información y la comunicación. Jóvenes y adultos usan con frecuencia Internet y la telefonía móvil (Instituto Nacional de Estadística, 2017), por lo que se hace preciso estudiar también la extensión del fenómeno del sexting a población adulta.

Los escasos estudios que se han interesado por analizar el sexting en otros grupos de mayor edad documentan una prevalencia entre el $12 \%$ y el $80 \%$ aproximadamente, dependiendo de la modalidad de sexting practicado. Este margen tan dispar en la prevalencia del sexting así como la insuficiencia de datos empíricos al respecto obliga a continuar estudiando el mismo en población adulta.

En la literatura científica revisada hasta el momento no se ha detectado ningún trabajo en España dedicado a estudiar el fenómeno en adultos. En el plano internacional, Ferguson (2011), desde Estados Unidos, informa de tasas de prevalencia en mujeres hispanas de 16 a 25 años en torno al 20\%. Gordon-Messer et al. (2013), también desde Estados Unidos, destacan que el 2\% de su muestra de jóvenes participantes (18-24 años) reconocía haber enviado contenidos de sexting, frente al $12.6 \%$ que decía haberlos recibido, y el $28.2 \%$ de la muestra indicaba que se había visto involucrado tanto en el envío como en la recepción de este tipo de contenidos. Por su parte, Morelli, Bianchi, Baiocco, Pezzuti y Chirumbolo (2016) encontraron que el $82.23 \%$ de los/as jóvenes italianos/as (13 - 30 años) informaba haber participado alguna vez en sexting, aunque indicaban que tales acciones no suponían un comportamiento frecuente o crónico al igual que han mostrado estudios previos (Weisskirch y Delevi, 2011). Del mismo modo, Morelli y su equipo (2016) hallaron que el $77.96 \%$ de participantes había recibido contenidos sexuales, el $63.12 \%$ había enviado este tipo de imágenes y el 8.77\% había posteado alguna vez imágenes íntimas, es decir, había publicado autoimágenes sexuales o eróticas en la red. Por consiguiente, al menos de lo que se desprende de estos estudios, parece que la participación más común en sexting tiene lugar a través de la recepción de contenidos de tipo sexual, más que a través del envío de estos contenidos a otras personas (Benotsch, Snipes, Martin y Bull, 2013; Gordon-Messer et al., 2013; Morelli et al., 2016).

Otras variables como las personas destinatarias del sexting, el género de los/as implicados/as y las motivaciones para el ejercicio de esta práctica, por su relevancia, requieren una mayor atención por parte de la comunidad científica. Algunos datos preliminares en este sentido parecen subrayar una tendencia a la práctica del sexting especialmente en el contexto de una relación de pareja (Drouin, Coupe y Temple, 2017; Yeung, Horyniack, Vella, Hellard y Lim, 2014). Por ejemplo, Drouin, Vogel, Surbey y Stills (2013) hallaron que el 49\% de los/as participantes universitarios/as había enviado una 
fotografía o vídeo sexual a su pareja estable, el $37 \%$ a personas con las que mantenía relaciones esporádicas y el $45 \%$ había realizado el envío en el contexto de una relación de infidelidad.

En cuanto a la variable género, en términos generales los datos con los que se cuenta hasta el momento parecen apuntar a una mayor prevalencia de hombres implicados, en comparación con las mujeres, tanto en el caso del ejercicio de esta conducta de forma moderada o altamente frecuente (Morelli et al., 2016). Al mismo tiempo, algunos trabajos sostienen que también son los hombres, en comparación con las mujeres, los que reciben en mayor medida imágenes de sexting y los que reenvían con mayor frecuencia este tipo de imágenes (Gordon-Messer et al., 2013). Sin embargo, otros estudios señalan que el envío de contenidos íntimos se lleva a cabo en mayor medida por parte de las mujeres (Wysocki y Childers, 2011). Resultados por el momento inconsistentes que señalan la necesidad de seguir profundizando en el estudio de la variable género relacionada con el sexting.

Por otra parte, al tratarse de un fenómeno reciente, variables cuyo conocimiento podría ser relevante para su mejor comprensión, como son las motivaciones para ejercer conductas de sexting, se han explorado escasamente. Se ha señalado el flirteo, la intención de iniciar relaciones sexuales (Drouin et al., 2013) y la coerción ejercida por otras personas (Drouin, Ross y Tobin, 2015; Englander, 2012) como los principales motivos encontrados para la práctica de sexting. En este sentido, se ha señalado que las mujeres podrían llevar a cabo conductas de sexting por presiones de sus parejas o de otros hombres (Walker, Sanci y Temple-Smith, 2013), mientras que los hombres usarían el sexting con mayor probabilidad para atraer a una posible pareja, y las mujeres para mantener sus relaciones actuales (Delevi y Weisskirch, 2013).

\subsection{El presente estudio}

Como se ha mencionado, la literatura científica se está centrando en la actualidad en estudiar el comportamiento de sexting en población adolescente, prestando menor atención a su análisis en otros grupos de mayor edad. Este novedoso trabajo tiene como objetivo analizar los comportamientos de sexting en una muestra de adultos españoles. En concreto, este estudio se centra en (1) conocer la prevalencia y cronicidad de sexting en una muestra de adultos, (2) determinar con qué personas intercambian los contenidos sexuales, (3) investigar las motivaciones para la práctica de sexting, y (4) analizar la influencia del género y la edad en este fenómeno.

Derivadas de los anteriores objetivos, se establecen las siguientes hipótesis de investigación: (1) existencia de comportamientos de sexting, tanto activos como pasivos, en la muestra de participantes, (2) el sexting se realiza especialmente con la pareja o en el contexto de una relación íntima, (3) las principales motivaciones para el sexting serán el flirteo o iniciar relación, mantener relaciones sexuales o debido a coerción ejercida por otra persona, y (4) se espera encontrar diferencias significativas en función del género y la edad en las variables incluidas en el estudio.

\section{Método}

\subsection{Participantes}

La muestra de estudio estuvo compuesta por 171 participantes de nacionalidad española seleccionados mediante muestreo no probabilístico y con edades comprendidas entre los 18 y 38 años de edad $(M=25.91 ; M e=26 ; D T=5.72)$. El porcentaje de mujeres fue del $63.7 \%(n=109)$ y el de hombres del $36.3 \%(n=62)$, mientras que el $91.8 \%$ de participantes manifestaban ser heterosexuales $(n=157)$. Esta muestra de participantes estaba compuesta por población general $(39.21 \%)$ y por población estudiante de formación profesional y formación universitaria $(60.79 \%)$.

\subsection{Instrumentos}

Para la recogida de información se diseñó un cuestionario que incluía las siguientes secciones e instrumentos de medida. 
Información sociodemográfica. Se recogieron datos referentes al sexo (Hombre vs. Mujer), edad, nacionalidad, orientación sexual y ocupación de los/as participantes.

Comportamientos de sexting. Seis ítems dirigidos a evaluar el comportamiento de sexting. En concreto, se evaluó: (1) Realización de selfies sexuales ( $\dot{c}$ Te has fotografiado y/o grabado a ti mismo en actitud provocativa o insinuante?); (2) Involucración en sexting activo (¿Has enviado imágenes provocativas o insinuantes de ti mismo/a a otra persona?); (3) Personas receptoras del sexting activo (Si has enviado imágenes de este tipo a alguien, ¿a quién se las has enviado?); (4) Motivaciones para involucrarse en sexting activo (En el caso de haber enviado tus imágenes íntimas a alguna persona, ¿por qué lo has hecho?); (5) Involucración en sexting pasivo (¿Has recibido imágenes provocativas o insinuantes que otra persona se ha tomado de sí misma?); (6) Personas emisoras en el sexting pasivo (Si has recibido imágenes íntimas que otra persona se ha tomado a sí misma, ¿de quién se trataba?). Los ítems (1), (2) y (5) presentan formato de respuesta tipo likert desde 1 (nunca) a 5 (muchas veces), mientras que los ítems (3), (4) y (6) muestran formato de respuesta de elección múltiple, siendo posible seleccionar varias de las opciones presentadas.

\subsection{Procedimiento}

La recogida de datos para este estudio se llevó a cabo mediante un cuestionario diseñado para tal fin en formato electrónico (aplicación proporcionada por la compañía Google). El hipervínculo o enlace a dicho formulario fue enviado a través de correo electrónico a un grupo de estudiantes de primer curso de Educación Primaria de la Universidad de Sevilla, solicitándoles a su vez el reenvío del enlace a sus contactos mayores de 18 años. Los/as participantes podían completar el cuestionario a través del teléfono móvil o de cualquier dispositivo electrónico con conexión a Internet. Las respuestas se almacenaban en la aplicación de Google, permitiendo su consulta y acceso a las investigadoras. Antes de cumplimentar el cuestionario, los/as participantes leían las instrucciones y se les informaba del carácter anónimo y confidencial de la información que iban a aportar. Una vez finalizado el cuestionario, las respuestas se guardaban automáticamente en la aplicación y se transfirieron los datos para ser analizados. Finalmente, se agradecía a los/as participantes su colaboración y se les pedía que enviasen el hipervínculo o enlace del formulario a sus contactos para que participasen.

\subsection{Análisis de datos}

Los datos obtenidos se analizaron mediante el programa estadístico SPSS (versión 24.0). Se realizaron análisis descriptivos de la variable edad de los y las participantes, y se dividió la muestra según la mediana obtenida. Esto permitió obtener dos grupos a partir de los cuales realizar comparaciones de las puntuaciones según las variables de interés en este estudio; un grupo de menor edad formado por participantes de 18 a 26 años, y otro grupo de mayor edad con participantes de 27 a 38 años. Para determinar la prevalencia de selfie sexual, sexting activo y sexting pasivo se llevaron a cabo análisis de frecuencias. También se empleó el análisis de frecuencias para determinar con qué personas se realizaba el sexting (personas receptoras de sexting activo y personas emisoras de sexting pasivo) y las motivaciones para involucrarse en sexting activo. Para examinar la cronicidad de tales conductas, se emplearon análisis descriptivos. Se utilizó la prueba Chi Cuadrado de Pearson como medida de contraste de las diferencias halladas en las distribuciones de frecuencias según la variable género y la variable edad, del mismo modo que se utilizó la prueba T de Student de muestras independientes para estudiar diferencias en la cronicidad del sexting. El tamaño del efecto se valoró atendiendo al valor $\mathrm{V}$ de Crámer y d de Cohen. El nivel de significación estadística requerido en las pruebas utilizadas fue de $p<0.05$. 


\section{Resultados}

3.1. Selfie sexual, Sexting activo y Sexting pasivo

Como muestra la Tabla 1, el 45\% de los/as participantes ha tomado en alguna ocasión una imagen de sí mismo de carácter sexual, aunque esta conducta no se realiza frecuentemente $(M=1.73$; $D . T=1.02)$. Por otro lado, el $40.9 \%$ ha realizado sexting activo, es decir, ha enviado una imagen íntima a otra persona, también de manera infrecuente en términos generales $(M=1.67 ; D . T=1.02)$. Por tanto, solo siete participantes han tomado imágenes de sí mismos pero no las han compartido con nadie. Igualmente, el $64.3 \%$ ha participado en sexting pasivo, esto es, ha recibido imágenes sexuales de otras personas (se descartan contenidos sexuales publicitarios y/o de la industria pornográfica), esta vez con mayor frecuencia que el sexting activo $(M=2.30 ; D . T=1.30)$.

En cuanto al género, se observan diferencias estadísticamente significativas entre hombres y mujeres en las conductas de sexting activo y sexting pasivo. En concreto, las diferencias se encuentran en la prevalencia de sexting activo $\left[\chi^{2}(1,171)=4.586, p<.05\right]$, que sería mayor en hombres que en mujeres, aunque el efecto observado es pequeño $(V=.164)$, y en la cronicidad de sexting pasivo $[t$ $(171)=-2.257, p<.05]$, que sería también superior en hombres frente a mujeres, con un tamaño del efecto pequeño-moderado $(d=.364)$.

En cuanto a la edad, se determinaron diferencias estadísticamente significativas en sexting pasivo, tanto en prevalencia $\left[\chi^{2}(1,171)=5.700, p<.05\right]$, con un efecto pequeño $(V=.183)$, como en cronicidad $[t(171)=2.675, p<.05]$, con un un tamaño del efecto pequeño-moderado $(d=.406)$. En ambos casos, es superior la implicación de los/as participantes menores de 26 años.

Tabla 1

Prevalencia y cronicidad de Selfie sexual, Sexting Activo y Sexting Pasivo según género y edad

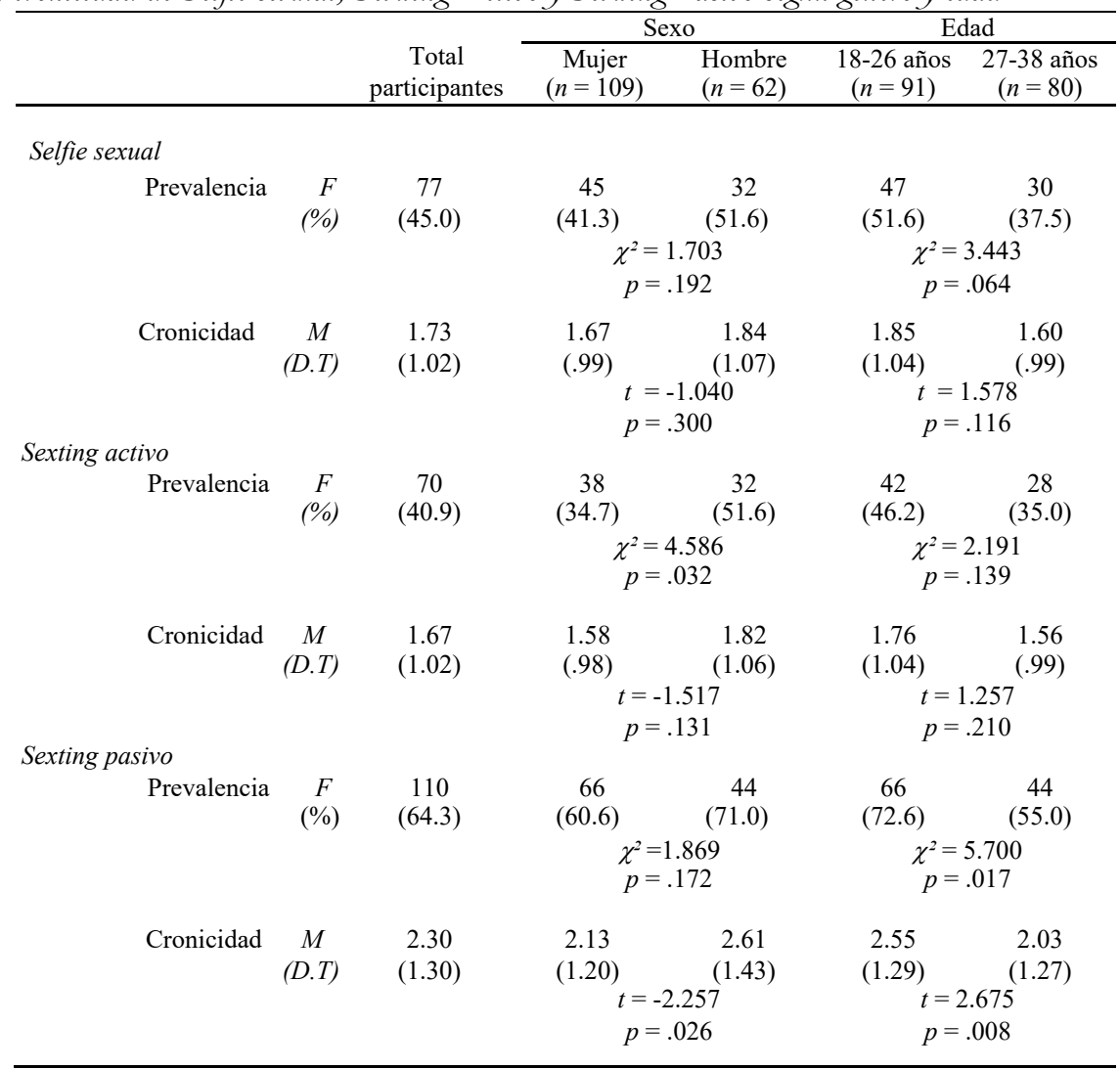

$F=$ Frecuencia $; \%)=$ Porcentaje $\chi^{2}=$ Estadístico Chi Cuadrado; $M=$ Media; $(D . T)=$ Desviación Típica; $t$ $=$ Estadístico $\mathrm{t}$ de Student 


\subsection{Personas receptoras y emisoras en el sexting}

En la Tabla 2 se muestran las personas involucradas en los intercambios de sexting. Las imágenes sexuales se envían más comúnmente a la pareja (34.5\%) y, en menor medida, a alguien que les atrae (8.8\%). Infrecuentemente se envían a alguien que se conoce de Internet $(2.3 \%)$ y nunca a una persona conocida diferente de las presentadas. Por otra parte, los/as participantes reciben las imágenes sexuales especialmente de sus parejas (38.6\%), y de personas que les atraen (15.2\%). En esta ocasión, sí se reciben contenidos de personas conocidas diferentes de los perfiles presentados $(11.7 \%)$, siendo menos frecuente recibir imágenes de contactos de Internet $(7.1 \%)$.

En sexting activo no se observaron diferencias estadísticamente significativas entre hombres y mujeres, aunque en sexting pasivo sí se evidenciaron, de tal forma que los hombres, en comparación con las mujeres, recibían con mayor frecuencia imágenes sexuales de alguien que les atrae $\left[\chi^{2}(1,171)\right.$ $=4.104, p<.05]$, un tamaño del efecto pequeño $(V=.155)$.

Por otro lado, se observaron puntuaciones diferenciales que resultaron significativas en función de la edad. El grupo de menor edad enviaban con mayor frecuencia imágenes íntimas a amistades $\left[\chi^{2}(1,171)=5.772, p<.05\right]$ y también las recibían con mayor frecuencia de sus amistades $\left[\chi^{2}(1,171)=4.572, p<.05\right]$. Además, recibían en mayor medida imágenes del sexting de alguien que les atrae frente al grupo de mayor edad $[\chi 2(1,171)=4.858, p<.05]$. La relación entre las variables que muestran estos tres casos resultó de intensidad baja $\left(V_{1}=.155 ; V_{2}=.164 ; V_{3}=.169\right)$.

Tabla 2.

Personas con las que se intercambian contenidos en el sexting (activo y pasivo) según género y edad

\begin{tabular}{|c|c|c|c|c|c|c|}
\hline & & $\begin{array}{l}\text { Pareja } \\
F(\%)\end{array}$ & $\begin{array}{c}\text { Amistades } \\
F(\%)\end{array}$ & $\begin{array}{c}\text { Alguien que } \\
\text { atrae } \\
F(\%)\end{array}$ & $\begin{array}{c}\text { Alguien de } \\
\text { Internet } \\
F(\%)\end{array}$ & $\begin{array}{c}\text { Otros } \\
\text { conocidos } \\
F(\%) \\
\end{array}$ \\
\hline Receptores & Total & $59(34.5 \%)$ & $10(5.8 \%)$ & $15(8.8 \%)$ & $4(2.3 \%)$ & 0 \\
\hline \multirow{7}{*}{ Sexting Activo } & Mujer & $33(30.3 \%)$ & $5(4.6 \%)$ & $8(7.3 \%)$ & $2(1.8 \%)$ & 0 \\
\hline & Hombre & $26(41.9 \%)$ & $5(8.1 \%)$ & $7(11.3 \%)$ & $2(3.2 \%)$ & 0 \\
\hline & & $\begin{aligned} \chi^{2} & =2.378 \\
n & =123\end{aligned}$ & $\begin{array}{c}\chi^{2}=.868 \\
n=352\end{array}$ & $\begin{aligned} \chi^{2} & =.771 \\
n & =380\end{aligned}$ & $\begin{array}{c}\chi^{2}=.335 \\
n=563\end{array}$ & - \\
\hline & 18-26 años & $36(39.6 \%)$ & $9(9.9 \%)$ & $8(8.8 \%)$ & $1(1.1 \%)$ & - \\
\hline & 27-38 años & $23(28.7 \%)$ & $1(1.3 \%)$ & $7(8.8 \%)$ & $3(3.8 \%)$ & - \\
\hline & & $\chi^{2}=2.202$ & $\chi^{2}=5.772$ & $\chi^{2}=.000$ & $\chi^{2}=1.310$ & - \\
\hline & & $p=.138$ & $p=.016$ & $p=.992$ & $p=.252$ & \\
\hline Emisores & Total & $66(38.6 \%)$ & $23(13.5 \%)$ & $26(15.2 \%)$ & $12(7.1 \%)$ & $20(11.7 \%)$ \\
\hline \multirow[t]{7}{*}{ Sexting Pasivo } & Mujer & $40(36.7 \%)$ & $17(15.6 \%)$ & $12(11.0 \%)$ & $9(8.3 \%)$ & $10(9.2 \%)$ \\
\hline & Hombre & $26(41.9 \%)$ & $6(9.7 \%)$ & $14(22.6 \%)$ & $3(4.8 \%)$ & $10(16.1 \%)$ \\
\hline & & $\chi^{2}=.458$ & $\chi^{2}=1.189$ & $\chi^{2}=4.104$ & $\chi^{2}=.708$ & $\chi^{2}=1.851$ \\
\hline & & $p=.499$ & $p=.275$ & $p=.043$ & $p=.400$ & $p=.174$ \\
\hline & $18-26$ años & $41(45.1 \%)$ & $17(18.7 \%)$ & $19(20.9 \%)$ & $4(4.4 \%)$ & $13(14.3 \%)$ \\
\hline & $27-38$ años & $25(31.3 \%)$ & $6(7.5 \%)$ & $7(8.8 \%)$ & $8(10.0 \%)$ & $7(8.8 \%)$ \\
\hline & & $\begin{aligned} \chi^{2} & =3.423 \\
p & =.064\end{aligned}$ & $\begin{aligned} \chi^{2} & =4.572 \\
p & =.032\end{aligned}$ & $\begin{aligned} \chi^{2} & =4.858 \\
p & =.028\end{aligned}$ & $\begin{aligned} \chi^{2} & =2.049 \\
p & =.152\end{aligned}$ & $\begin{aligned} \chi^{2} & =1.263 \\
p & =.261\end{aligned}$ \\
\hline
\end{tabular}

$F=$ Frecuencia; $(\%)=$ Porcentaje $; \chi^{2}=$ Estadístico Chi Cuadrado.

\subsection{Motivaciones para realizar sexting}

La Tabla 3 presenta los motivos para realizar sexting activo informados en este estudio. El $19.3 \%$, de los/as participantes indicó que practicaba sexting con el objetivo de satisfacer a la persona receptora de los contenidos, mientras que el 17\% indicaba que lo hacía por bromear. El 1.2\% de participantes aducía como motivación para enviar contenidos sexuales otras razones diferentes y el $0.6 \%$, por obligación. Solamente una participante indicó haber realizado sexting por coerción. Los análisis efectuados no mostraron diferencias estadísticamente significativas en función del género, 
aunque sí en función de la edad. Los participantes de menor edad informaron que realizaban sexting con la intención de mantener una relación sexual en mayor medida que los de mayor edad $\left[\chi^{2}(1\right.$, $171)=8.296, p<.05]$, observándose en este caso un tamaño del efecto pequeño $(V=.220)$.

Tabla 3

Motivos para realizar sexting activo según género y edad

\begin{tabular}{|c|c|c|c|c|c|c|c|c|c|}
\hline \multirow[b]{2}{*}{ Motivos } & \multirow[b]{2}{*}{$\begin{array}{c}\text { Total } \\
F(\%) \\
\end{array}$} & \multicolumn{2}{|c|}{ Género } & \multicolumn{6}{|c|}{ Edad } \\
\hline & & $\begin{array}{l}\text { Mujer } \\
F(\%)\end{array}$ & $\begin{array}{c}\text { Hombre } \\
F(\%)\end{array}$ & $\chi^{2}$ & $p$ & $\begin{array}{c}18-26 \text { años } \\
F(\%)\end{array}$ & $\begin{array}{c}27-38 \text { años } \\
F(\%)\end{array}$ & $\chi^{2}$ & $p$ \\
\hline Satisfacer & $\begin{array}{c}33 \\
(19.3 \%)\end{array}$ & $\begin{array}{c}17 \\
(15.6 \%)\end{array}$ & $\begin{array}{c}16 \\
(25.8 \%)\end{array}$ & 2.645 & .104 & $\begin{array}{c}20 \\
(22.0)\end{array}$ & $\begin{array}{c}13 \\
(16.3)\end{array}$ & .897 & .344 \\
\hline Gustar & $\begin{array}{c}21 \\
(12.3 \%)\end{array}$ & $\begin{array}{c}15 \\
(13.8 \%)\end{array}$ & $\begin{array}{c}6 \\
(9.7 \%)\end{array}$ & .612 & .434 & $\begin{array}{c}14 \\
(15.4)\end{array}$ & $\begin{array}{c}7 \\
(8.8)\end{array}$ & 1.740 & .187 \\
\hline Relación Sexual & $\begin{array}{c}22 \\
(12.9 \%)\end{array}$ & $\begin{array}{c}13 \\
(11.9 \%)\end{array}$ & $\begin{array}{c}9 \\
(14.5 \%)\end{array}$ & .236 & .627 & $\begin{array}{c}18 \\
(19.8)\end{array}$ & $\begin{array}{c}4 \\
(5.0)\end{array}$ & 8.296 & .004 \\
\hline Iniciar relación & $\begin{array}{c}2 \\
(1.2 \%)\end{array}$ & $\begin{array}{c}1 \\
(0.9 \%)\end{array}$ & $\begin{array}{c}1 \\
(1.6 \%)\end{array}$ & .165 & .684 & 0 & $\begin{array}{c}2 \\
(2.5)\end{array}$ & 2.302 & .129 \\
\hline Bromear & $\begin{array}{c}29 \\
(17.0 \%)\end{array}$ & $\begin{array}{c}17 \\
(15.6 \%)\end{array}$ & $\begin{array}{c}12 \\
(19.4 \%)\end{array}$ & . 396 & .529 & $\begin{array}{c}19 \\
(20.9)\end{array}$ & $\begin{array}{c}10 \\
(12.5)\end{array}$ & 2.122 & .145 \\
\hline Coerción & $\begin{array}{c}1 \\
(0.6 \%)\end{array}$ & $\begin{array}{c}1 \\
(0.9 \%)\end{array}$ & 0 & .572 & .449 & $\begin{array}{c}1 \\
(1.1)\end{array}$ & 0 & .884 & .347 \\
\hline Otras razones & $\begin{array}{c}2 \\
(1.2 \%)\end{array}$ & $\begin{array}{c}1 \\
(0.9 \%)\end{array}$ & $\begin{array}{c}1 \\
(1.6 \%)\end{array}$ & .165 & .684 & $\begin{array}{c}1 \\
(1.1)\end{array}$ & $\begin{array}{c}1 \\
(1.2)\end{array}$ & .008 & .927 \\
\hline
\end{tabular}

$F=$ Frecuencia; $(\%)=$ Porcentaje; $\chi^{2}=$ Estadístico Chi Cuadrado.

\section{Discusión}

Este trabajo analiza por vez primera comportamientos de sexting en una muestra de jóvenes adultos españoles con el objetivo de conocer la prevalencia y las motivaciones para su práctica, así como posibles diferencias en función del género y de la edad. En general, los resultados muestran apoyo a las principales hipótesis del estudio y ponen de relieve que el sexting es una práctica frecuente en la población de adultos estudiada.

Los hallazgos muestran apoyo a la primera hipótesis de este trabajo relacionada con la prevalencia y frecuencia de los comportamientos de sexting. Así, los resultados muestran alta prevalencia y frecuencia de este tipo de prácticas en los adultos jóvenes participantes, porcentajes superiores que los hallados en el trabajo de Gordon-Messer et al. (2013) en población estadounidense aunque inferiores a los indicados por Morelli et al. (2016) en población italiana. Así, el 40.9\% de los participantes ha enviado imágenes íntimas a otra persona a través de las tecnologías, y que el $64.3 \%$ ha recibido imágenes sexuales de otras personas en sus dispositivos electrónicos. Por tanto, sería más prevalente y habitual recibir fotografías o vídeos íntimos que otra persona se ha realizado a sí misma que enviar imágenes propias de carácter sexual.

Los resultados también respaldan la segunda hipótesis de investigación, ya que el sexting en esta muestra de participantes tiene lugar especialmente con la pareja o en el curso de una relación íntima, resultados en línea con la literatura previa (Drouin et al., 2013; Drouin et al., 2017; Yeung et al., 2014). Las evidencias indican que el intercambio de imágenes sexuales (envío y recepción) es más habitual con la pareja frente a otro tipo de personas, como alguien que les atrae, amistades o contactos 
de Internet. Por consiguiente, parece que los contenidos sexuales se muestran a personas con las que se presume la existencia de un vínculo de intimidad y cercanía, al menos, en ese momento, lo que no implica necesariamente que dicha confianza y lealtad permanezca invariable en el tiempo.

En cuanto a las motivaciones para realizar sexting, se hipotetizó que los participantes indicarían el flirteo, iniciar una relación, mantener relaciones sexuales o por el ejercicio de coerción por parte de otra persona, en consonancia con investigaciones previas (Drouin et al., 2013; Drouin et al., 2015; Englander, 2012). Sin embargo, los resultados de este trabajo indican que en este grupo de jóvenes adultos sería poco común participar en sexting activo por coerción. Solo una participante indicó que había enviado imágenes sexuales propias por presiones, en este caso, una mujer de 19 años estudiante universitaria. Además, los resultados muestran que el sexting se realizaba principalmente con el propósito de satisfacer a la otra persona y para bromear. Una interpretación preliminar a estos datos podría sugerir que las personas que envían contenidos propios de carácter sexual priorizarían las necesidades del destinatario o banalizarían tales prácticas en vez de contemplar el riesgo que asumen al poner en circulación contenidos de este tipo en la red.

En lo relativo a diferencias en función del género y la edad de los participantes, los resultados muestran un apoyo parcial a la cuarta hipótesis de este estudio. En cuanto al género, un mayor porcentaje de hombres que de mujeres reconocía haber enviado en alguna ocasión contenidos íntimos propios. Estos resultados estarían en consonancia con lo sostenido por Gordon-Messer y colaboradores (2013), pero irían en contra de lo hallado por Wysocki y Childers (2011), ya que las mujeres no serían las principales generadoras de contenidos sexuales. No obstante, los resultados ponen de manifiesto que no hay diferencias entre hombres y mujeres en cuanto a la prevalencia en sexting pasivo, aunque los hombres, en comparación con las mujeres, sí reciben con mayor frecuencia imágenes sexuales. Es decir, en esta muestra de participantes es más común encontrar que más hombres que mujeres hayan enviado imágenes eróticas suyas a otras personas, y que reciban con mayor frecuencia contenidos de este tipo en sus dispositivos electrónicos.

Continuando con la hipótesis cuarta, las evidencias muestran que hombres y mujeres envían sus informaciones privadas con mayor frecuencia a sus parejas, aunque son los hombres participantes los que reciben en mayor medida imágenes sexuales de personas que les atraen pero con la que no mantienen una relación de pareja estable, es decir, contemplan este tipo de prácticas con personas con las que presumiblemente no les une una relación de intimidad y proximidad, lo que a priori podría parecer más arriesgado.

Por último, no se encontraron diferencias de género en los motivos para realizar sexting, aunque como se ha mencionado con anterioridad la única persona que indicó sentirse obligada a realizarlo por coerción fue una mujer joven.

En cuanto a diferencias en función de la edad, el sexting pasivo sería más prevalente y crónico en los adultos menores de 26 años frente a los del grupo de mayor edad, aunque parece ser que en esta muestra la participación activa en sexting es independiente de la edad. Por tanto, enviar imágenes de uno mismo en actitud provocativa no sería un comportamiento exclusivo en edades tempranas. Serían conductas observables también en la edad adulta. Asimismo, los participantes menores de 26 años son los que reciben en mayor medida contenidos íntimos de personas que les atraen y quienes además reciben y envían imágenes de sexting a amistades de forma más habitual que los de mayor edad. Practicar sexting con la intención de mantener relaciones sexuales fue una motivación más frecuente en participantes de menor edad, aunque como se ha mencionado, no fue uno de los principales motivos indicados.

Esta investigación, por su carácter innovador, ha aportado datos interesantes sobre la práctica del sexting en una muestra de jóvenes adultos. No obstante, conviene señalar algunas limitaciones del estudio que podrían superarse en futuras investigaciones y que permitirían aumentar su potencia estadística y capacidad de generalización. La primera de ellas tiene que ver con el tamaño muestral que, aunque es suficiente para un estudio exploratorio, necesitaría aumentarse para obtener mayor confianza en la generalización de los resultados. Futuros trabajos podrían ampliar la muestra de estudio y proceder con un muestreo probabilístico, así como equilibrar el número de mujeres y hombres participantes y las edades contempladas. Igualmente, habría sido interesante analizar el tipo 
de relación de pareja que los y las participantes mantienen con quienes intercambian sus fotografías o vídeos íntimos (duración de la relación, existencia o no de convivencia, parejas formalizadas o no, infidelidad, etc.). Futuros estudios podrían analizar estas variables y profundizar en el análisis de las implicaciones que el uso de las tecnologías tiene en las formas relacionales en diversas etapas del ciclo vital, con especial atención a comportamientos de tipo sexual.

Estos resultados señalan la necesidad de incluir no solo a adolescentes en campañas educativas sobre sexting, sino también a mayores de edad. Estas acciones formativas son fundamentales para poner en relieve los riesgos potenciales de prácticas de este tipo y subrayar la necesidad de un uso seguro de los medios electrónicos. Tal y como sostienen González y Pérez (2010), la salud sexual también engloba la habilidad para comprender y evaluar los riesgos, responsabilidades, resultados e impacto de las actividades sexuales. Por tanto, se requiere una labor pedagógica más intensa y extensa para sensibilizar sobre las posibles complicaciones que podrían derivarse de un uso incorrecto de informaciones de tipo íntimo y privado, como podría ser la difusión de los contenidos sexuales a través de medios electrónicos.

En conclusión, este estudio pionero analiza el fenómeno del sexting en una muestra de jóvenes adultos españoles, aportando datos sobre su prevalencia, motivaciones y diferencias de género en tales comportamientos. Investigaciones previas se han centrado fundamentalmente en el análisis de este fenómeno en población adolescente, sin embargo esta investigación analiza tales prácticas en una muestra de adultos españoles poniendo de relieve una considerable prevalencia en el envío y recepción de imágenes íntimas de contenido sexual. Estas evidencias resaltan lo conveniente de continuar con la comprensión del sexting en mayores de edad así como de incluir a los mismos en las campañas de información y sensibilización de comportamientos de riesgo en la red.

\section{Referencias bibliográficas}

Alonso-Ruido, P., Rodríguez-Castro, Y., Pérez-André, C., y José-Magalhães, M. J. (2015). Estudio cualitativo en un grupo de estudiantes ourensanos/as sobre el fenómeno del sexting. Revista de Estudios e Investigación en Psicología y Educación, 2014(14), 10-14. doi: 10.17979/reipe.2015.0.

Benotsch, E., Snipes, D., Martin, A., y Bull, S. (2013). Sexting, substance use, and sexual risk behavior in young adults. Journal of Adolescent Health, 52(3), 307-313. doi: 10.1016/i.jadohealth.2012.06.011.

Cornejo, M., y Tapia, M. L. (2011). Redes sociales y relaciones interpersonales en internet. Fundamentos en Humanidades, 12(24), 219-229.

Cruz, L., y Soriano, E. (2014). Psychological aspects, attitudes and behaviour related to the practice of sexting: a systematic review of the existent literature. Procedia - Social and Behavioral Sciences, 132(132), 114-120. doi: 10.1016/j.sbspro.2014.04.286.

Dake, J. A., Price, D. H., Maziarz, L., y Ward, B. (2012). Prevalence and correlates of sexting behaviour in adolescents. American Journal of Sexuality Education, 7, 1-15. doi: 10.1080/15546128.2012.650959.

Delevi, R., y Weisskirch, R. S. (2013). Personality factors as predictors of sexting. Computers in Human Behavior, 29(6), 2589-2594. doi: 10.1016/j.chb.2013.06.003.

Drouin, M., Coupe, M., y Temple, J. R. (2017). Is sexting good for your relationship? It depends... Computers in Human Behavior, 75, 749-756. doi: 10.1016/j.chb.2017.06.018.

Drouin, M., Ross, J., y Tobin, E. (2015). Sexting: A new, digital vehicle for intimate partner aggression? Computers In Human Behavior, 50, 197-204. doi: 10.1016/j.chb.2015.04.001.

Drouin, M., Vogel, K. N., Surbey, A., y Stills, J. R. (2013). Let's talk about sexting, baby: Computermediated sexual behaviors among young adults. Computers in Human Behavior, 29(5), A25A30. doi: 10.1016/j.chb.2012.12.030. 
Durán, M. y Rodríguez-Domínguez, C. (2018). Nuevos tiempos, nuevos peligros: intercambio de contenidos sexuales entre adolescentes en la era digital. En A. Salvador, M. I. Iglesias, A. Picornell, J. J. Leiva, A. Pantoja, M. A. Conde (Coord.) Participación, politicas sociales y protección a la infancia (pp. 200-209). Sevilla, España: CIPI Ediciones.

Englander, E. (2012). Low risk associated with most teenage sexting: A study of 617 18- year-olds. Massachusetts Aggression Reduction Center. Consulta 5 de marzo 2018. Disponible en: https://bit.ly/2NkJufT.

Fajardo, M. I., Gordillo, M., y Regalado, A. B. (2013). Sexting: Nuevos usos de la tecnología y sexualidad en adolescentes. International Journal of Developmental and Education Psychology, 1(0214-9877), 521-534.

Ferguson, C. J. (2011). Sexting behaviors among young hispanic women: Incidence and association with other high-risk sexual behaviors. Psychiatric Quarterly, 82(3), 239-243. doi: 10.1007/s11126-010-9165-8

Gámez-Guadix, M., Santisteban, P., y Resett, S. (2017). Sexting entre adolescentes españoles: Prevalencia y asociación con variables de personalidad. Psicothema, 29(1), 29-34. doi: 10.7334/psicothema2016.222

González, A. J., y Pérez, G. (2010). Cultura, sexualidad y juventud: experiencias de nuestros jóvenes en Londres. Revista Fuentes (10), 154-172.

Gordon-Messer, D., Bauermeister, J. A., Grodzinski, A., y Zimmerman, M. (2013). Sexting among young adults. Journal of Adolescent Health, 52(3), 301-306. doi: 10.1016/i.jadohealth.2012.05.013.

Hinduja, S., y Patchin, J.W. (2012). School climate 2.0: Preventing cyberbullying and sexting one classroom at a time. Thousand Oaks, CA: Corwin Press.

Instituto Nacional de Estadística (2017). Encuesta sobre equipamiento y uso de tecnologías de información y comunicación en los hogares. Consulta 5 de marzo 2018. Disponible en: https://bit.ly/1LJt9dw.

Lenhart, A. (2009). Teens and Sexting. How and why minor teens are sending sexually suggestive nude or nearly nude images via text massaging. Pew Internet \& American Life Project. Consulta 4 de marzo 2018. Disponible en: https://pewrst.ch/2LqVDNV.

Mclaughlin, J. H. (2010). Crime and Punishment: Teen Sexting in Context. Penn State Law Review, 115(1), 135-181.

Menjívar, M. (2010).El sexting y 1@s nativ@s neo- tecnológic@s: apuntes para una contextualización al inicio del siglo XXI. Actualidades Investigativas en educación, 10(2), 1-23. 10.15517/aie.v10i2.10117.

Morelli, M., Bianchi, D., Baiocco, R., Pezzuti, L., y Chirumbolo, A. (2016). Sexting, psychological distress and dating violence among adolescents and young adults. Psicothema, 28(2), 137-142. $10.7334 /$ psicothema2015.193.

Pérez, P., Flores, J., De la Fuente, S., Álvarez, E., García, L., y Gutiérrez, C. (2011). Guía sobre adolescencia y sexting: qué es y cómo prevenirlo. Instituto Nacional de Tecnologías de la Comunicación (INTECO) y France Telecom España (Orange). Consulta 2 de marzo 2018. Disponible en: https://bit.ly/1ZlXKkp.

Rodríguez-Castro, Y., Alonso-Ruido, P., González-Fernández, A., Lameiras-Fernández, M., y Carrera-Fernández, M. V. (2017). Spanish adolescents' attitudes towards sexting: Validation of a scale. Computers in Human Behavior, 73, 375-384. 10.1016/j.chb.2017.03.049.

Van Ouytsel, J., Walrave, M., Ponnet, K., y Heirman, W. (2015). The association between adolescent sexting, psychosocial difficulties, and risk behavior. The Journal of School Nursing, 31(1), 54-69. $10.1177 / 1059840514541964$.

Walker, S., Sanci, L., y Temple-Smith, M. (2013). Sexting: Young women's and men's views on its nature and origins. Journal of Adolescent Health, 52(6), 697-701. 10.1016/i.jadohealth.2013.01.026.

Weisskirch, R., y Delevi, R. (2011). 'Sexting' and adult romantic attachment. Computers in Human Behavior, 27(5), 1697-1701. 10.1016/j.chb.2011.02.008. 
Wysocki, D., y Childers, C. D. (2011). Let my fingers do the talking: Sexting and infidelity in cyberspace. Sexuality \& Culture: An Interdisciplinary Quarterly, 15, 217-239. 10.1007/s12119011-9091-4.

Yeung, T., Horyniak, D., Vella, A., Hellard, M., y Lim, M. (2014). Prevalence, correlates and attitudes towards sexting among young people in Melbourne, Australia. Sexual Health, 11(4), 332-339. $10.1071 /$ SH14032.

\section{Financiación}

Este trabajo ha recibido financiación de la Unidad para la Igualdad (Universidad de Sevilla). Sevilla. España. Proyecto financiado: "Estudio sobre la ciberviolencia sexual en población joven universitaria de la universidad de Sevilla" (REF. 9332). Convocatoria 2017 de ayudas para la realización de iniciativas en materia de Igualdad de Género.

\section{Para citar este artículo}

Rodríguez-Domínguez, C. \& Durán Segura, M. (2019). Conductas sexuales de riesgo en la era digital: análisis del fenómeno sexting en la población adulta joven española. Revista Fuentes, 21(1), 3949. [Fecha de consulta: dd/mm/aa]. doi: 10.12795/revistafuentes.2019.v21.i1.03 